\title{
Lipid composition and abundance in the reproductive and alimentary tracts of female Haemonchus contortus
}

Tao Wang ${ }^{1}$ (D, Guangxu Ma1', Shuai Nie², Nicholas A. Williamson², Gavin E. Reid ${ }^{3,4,5}$ and Robin B. Gasser ${ }^{\text {* }}$

\begin{abstract}
Background: Lipids play essential structural and functional roles in the biology of animals. Studying the composition and abundance of lipids in parasites should assist in gaining a better understanding of their molecular biology, biochemistry and host-parasite interactions.

Methods: Here, we used a combination of high-performance liquid chromatography and mass spectrometric analyses, combined with bioinformatics, to explore the lipid composition and abundance in the reproductive (Rt) and alimentary (At) tracts of Haemonchus contortus.

Results: We identified and quantified 320 unique lipid species representing four categories: glycerolipids, glycerophospholipids, sphingolipids and steroid lipids. Glycerolipids (i.e. triradylglycerols) and glycerophospholipids (i.e. glycerophosphocholines) were the most commonly and abundant lipid classes identified and were significantly enriched in Rt and At, respectively. We propose that select parasite-derived lipids in Rt and At of adult female $H$. contortus are required as an energy source (i.e. triradylglycerol) or are involved in phospholipid biosynthesis (i.e. incorporated fatty acids) and host-parasite interactions (i.e. phospholipids and lysophospholipids).

Conclusions: This work provides a first foundation to explore lipids at the organ-specific and tissue-specific levels in nematodes, and to start to unravel aspects of lipid transport, synthesis and metabolism, with a perspective on discovering new intervention targets.
\end{abstract}

Keywords: Haemonchus contortus, Reproductive tract, Gut, Parasitic nematode, Lipidome, Lipids, Mass spectrometry, Adaptation

\section{Background}

Haemonchus contortus (the barber's pole worm) is one of the most pathogenic parasitic nematodes of ruminants, causing haemonchosis, which leads to major productivity and financial losses to agricultural and associated industries worldwide [1, 2]. Control relies heavily on chemotherapeutic treatment and/

\footnotetext{
*Correspondence: robinbg@unimelb.edu.au

${ }^{1}$ Department of Veterinary Biosciences, Melbourne Veterinary

School, Faculty of Veterinary and Agricultural Sciences, The University of Melbourne, Parkville, Victoria 3010, Australia

Full list of author information is available at the end of the article
}

or vaccination [3]. However, due to the excessive and often uncontrolled usage of anthelmintics, $H$. contortus has developed resistance to most drug classes in current use [4], even to the recently-introduced aminoacetonitrile derivate, monepantel [5]. Despite the utility of vaccination in some age groups of animals (usually sheep) [1], there is a need to pursue the discovery of new interventions against haemonchosis. We believe that a profound understanding of the molecular biology of $H$. contortus could assist in the discovery of novel drug and vaccine targets in this worm.

In the past decade, progress has been made in the genomics, transcriptomics, proteomics,

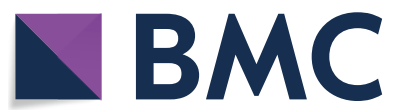

(c) The Author(s) 2020. This article is licensed under a Creative Commons Attribution 4.0 International License, which permits use, sharing, adaptation, distribution and reproduction in any medium or format, as long as you give appropriate credit to the original author(s) and the source, provide a link to the Creative Commons licence, and indicate if changes were made. The images or other third party material in this article are included in the article's Creative Commons licence, unless indicated otherwise in a credit line to the material. If material is not included in the article's Creative Commons licence and your intended use is not permitted by statutory regulation or exceeds the permitted use, you will need to obtain permission directly from the copyright holder. To view a copy of this licence, visit http://creativeco mmons.org/licenses/by/4.0/. The Creative Commons Public Domain Dedication waiver (http://creativecommons.org/publicdomain/ zero/1.0/) applies to the data made available in this article, unless otherwise stated in a credit line to the data. 
phosphoproteomics and lipidomics of this nematode via next generation nucleic acid sequencing or high throughput mass spectrometry approaches [6-13]. Studies are providing new and exciting insights into essential biological processes in key developmental stages of $H$. contortus, and are paving a way to identifying potential intervention targets, such as the bile acid-like hormone signalling receptors and cholesterol transporters (e.g. [14]). We believe that the study of lipids and their biology could also reveal intervention targets.

Recently, using a high throughput LC-MS/MS approach, we characterised the lipidome of different developmental stages and both sexes of $H$. contortus, and showed a substantial down-regulation of energy storage-related lipids, i.e. triradylglycerols (TG), during the transition from free-living to parasitic stages, suggesting critical and specific adaptations of the nematode (e.g. shifting or switching of nutrient acquisition) during its life-cycle [11]. Interestingly, TG abundance was relatively high in female adults of $H$. contortus compared with male adults. Considering the high abundance of total TG in eggs released by $H$. contortus, we proposed that immature eggs in the uterus represent a major proportion of TG in female worms. As nematodes, including Ascaris, convert enzymatically-released fatty acids into direct energy supply for egg development [15], it seems likely that $H$. contortus females actively accumulate energy as fat in embryos, to prepare them for life in the external environment. Logically extending previous work [15], we undertook the present study to gain a deeper insight into lipid composition and abundance in the reproductive and alimentary systems of the adult female of $H$. contortus.

\section{Methods}

\section{Haemonchus contortus and its procurement}

Adult worms of $H$. contortus were produced in sheep [16]. Worms were collected after 21 days of infection, washed five-times in large volumes $(100 \mathrm{ml})$ of physiological saline ( $\mathrm{pH} 7.0$ ), and gravid female worms with a length of $>2 \mathrm{~cm}$ were selected. The reproductive (Rt) and alimentary (At) tracts were dissected from these female worms using a dissecting microscope $(10 \times$ magnification), and snap-frozen at $-80{ }^{\circ} \mathrm{C}$; whole gravid female worms (Wf; reference control) were frozen in the same manner. Four replicates were prepared for each Rt, At and Wf.

\section{Tissue homogenisation and lipid extraction}

All four replicate samples of each Rt, At and Wf were individually homogenised and processed using an established method [11] and then lyophilised in a benchtop, manifold freeze-drier prior to extraction. Freeze-dried samples (2 mg each) were individually transferred to an
Eppendorf tube $(1.5 \mathrm{ml})$ containing $200 \mu \mathrm{l}$ of ice-cold $40 \%(\mathrm{v} / \mathrm{v})$ methanol. Samples were homogenised with $100 \mu \mathrm{l}$ of $0.5 \mathrm{~mm}$ zirconium oxide beads (ZROB05, Next Advance, USA) in a blender (Bead Bullet, Next Advance, USA) twice for $3 \mathrm{~min}$. Blank tubes with water (included as controls) were processed in the same manner. Lipids were extracted using an established method [17]. Briefly, $400 \mu \mathrm{l}$ of chloroform/methanol $(2 / 1, \mathrm{v} / \mathrm{v})$ were added to each tube. The tubes were vortexed for $60 \mathrm{sec}$, and centrifuged at $3000 \times g$ and $22{ }^{\circ} \mathrm{C}$ for $10 \mathrm{~min}$. The lower organic phase was then transferred to another tube, and $400 \mu \mathrm{l}$ of $100 \%$ chloroform were added, vortexed (60 s) and centrifuged as before. The lower organic phase was collected and pooled with the organic phase from the first extraction. The pooled lipid samples were then dried in a SpeedyVac $(4000 \times g$ for $45 \mathrm{~min})$, and each lipid pellet was re-suspended in $10 \mu \mathrm{l}$ isopropanol/methanol/chloroform $(4 / 2 / 1, \mathrm{v} / \mathrm{v} / \mathrm{v})$ containing $0.01 \%$ butylated hydroxytoluene (BHT), and then diluted with $190 \mu \mathrm{l} 100 \%$ methanol prior to analysis.

\section{High-performance liquid chromatography (HPLC) and mass spectrometric (MS) analyses}

All three samples (four replicates for each) were analysed by ultrahigh performance liquid chromatography (UHPLC) coupled to tandem mass spectrometry (MS/ MS) employing a Vanquish UHPLC linked to an Orbitrap Fusion Lumos mass spectrometer (Thermo Fisher Scientific, San Jose, CA, USA), with separate runs in positive and negative ion polarities. Solvent $A$ was $6 / 4(\mathrm{v} / \mathrm{v})$ acetonitrile/water and solvent B was $9 / 1(\mathrm{v} / \mathrm{v})$ isopropanol/acetonitrile; both solvents A and B contained 10 $\mathrm{mM}$ ammonium acetate. Each sample (volume: $4 \mu \mathrm{l}$ ) was injected into an Accucore C30 column $(2.1 \times 250 \mathrm{~mm}$, $2.6 \mu \mathrm{m}$; Thermo Fisher Scientific) at $40{ }^{\circ} \mathrm{C}$ at a flow rate of $350 \mu \mathrm{l} / \mathrm{min}$ for $1 \mathrm{~min}$ using 30\% solvent B. During separation, the percentage of solvent $B$ was increased from $30 \%$ to $70 \%$ in $5 \mathrm{~min}$, from $70 \%$ to $93 \%$ in $9 \mathrm{~min}$, from $93 \%$ to $99 \%$ in $7 \mathrm{~min}$, and from $91 \%$ to $97 \%$ in $31 \mathrm{~min}$. Subsequently, the percentage of solvent B was increased to $99.5 \%$ in $0.1 \mathrm{~min}$ and then maintained at $99.5 \%$ for 4.9 min. Finally, the percentage of solvent $B$ was decreased to $30 \%$ in $0.1 \mathrm{~min}$ and maintained for $3.9 \mathrm{~min}$.

All MS experiments were performed using a Heated Electrospray Ionization (HESI) source. The spray voltages were $3.5 \mathrm{kV}$ in positive ionisation-mode and 3.0 $\mathrm{kV}$ in negative ionisation-mode. In both polarities, the flow rates of sheath, auxiliary and sweep gases were 20 and 6 and 1 'arbitrary' units, respectively. The ion transfer tube and vaporizer temperatures were maintained at $350{ }^{\circ} \mathrm{C}$ and $400{ }^{\circ} \mathrm{C}$, respectively, and the $\mathrm{S}$-Lens RF level was set at $50 \%$. In the positive ionisation-mode from 1 to $28 \mathrm{~min}$, top speed data-dependent 
scan with a cycle time of $1 \mathrm{~s}$ was used. Within a cycle, a full-scan MS spectra were acquired firstly in the Orbitrap at a mass resolving power of 120,000 (at m/z 200) across an $\mathrm{m} / \mathrm{z}$ range of 300-2000 using quadrupole isolation, an automatic gain control (AGC) target of $4 \mathrm{e} 5$ and a maximum injection time of $50 \mathrm{msec}$. Then, every higher-energy collisional dissociation (HCD)MS/MS was performed in the cycle at a mass resolving power of 15,000 (at $\mathrm{m} / \mathrm{z} 200$ ), a normalised collision energy (NCE) of $27 \%$, an $\mathrm{m} / \mathrm{z}$ isolation window of 1 , a maximum injection time of 35 milliseconds and an AGC target of 5e4. For the improved structural characterisation of glycerophosphocholine (PC) lipid ions, a data-dependent product ion $(\mathrm{m} / \mathrm{z} 184.0733)$-triggered collision-induced dissociation (CID)-MS/MS scan was performed in the cycle using a q-value of 0.25 and a NCE of $30 \%$, with other settings being the same as that for HCD MS/MS. For the improved structural characterisation of TG lipids, the fatty acid + NH3 neutral loss product ions observed by HCD-MS/MS were used to trigger the acquisition of the top-3 data-dependent CID-MS3 scans in the cycle using a q-value of 0.25 and a NCE of $30 \%$, with other settings being the same as that for HCD MS/MS.

\section{Identification and quantification of lipids and statistical analysis}

MS data were processed using LipidSearch software v.4.2.23 (Thermo Fisher Scientific, San Jose, CA, USA) [18]. Key processing parameters were: target database, general; precursor tolerance, $5 \mathrm{ppm}$; product tolerance, 5 ppm; product ion threshold, $1 \%$; $\mathrm{m}$-score threshold, 2 ; quantification $\mathrm{m} / \mathrm{z}$ tolerance, $\pm 5 \mathrm{ppm}$; quantification retention time range, $\pm 1 \mathrm{~min}$; use of main isomer filter and ID quality filters $\mathrm{A}, \mathrm{B}$ and $\mathrm{C}$; adduct ions, $+\mathrm{H}$ and $+\mathrm{NH} 4$ for positive ionisation mode, and $-\mathrm{H}$ and $+\mathrm{CH} 3 \mathrm{COO}$ for negative ionisation mode. All lipid classes available were selected for the search. The same lipid annotations (within $\pm 0.1 \mathrm{~min}$ ) were merged into the aligned results. Unassigned peak areas were calculated for relative quantification and alignment. The shorthand notation used for lipid classification and structural representation follows the nomenclature proposed previously $[19,20]$. To filter false identifications, aligned lipids were examined manually. For TG lipids, a grade $\mathrm{A}$-identification required at least one replicate of each sample, whereas a grade A-, B- or C-identification required only monoacylglycerol (MG) lipids. For all other lipid classes, a grade A- or B-identification required at least one replicate of each sample. For all lipids, a signal-to-noise ratio $(\mathrm{S} / \mathrm{N})>10$ and a peak area $>1 \mathrm{e} 7$ were required in at least one sample group. All lipid LC-MS features were manually inspected and reintegrated when needed. To compare the relative abundance of lipids between tracts (tissues) and the whole worm of $H$. contortus, the ion abundances of corresponding lipid categories and classes were expressed as a percentage of the total ion abundance for the total lipidome, whereas the ion abundances of corresponding lipid species were expressed as a percentage of the total ion abundance for a particular lipid class. Venn diagrams of individual lipidomes were produced using the $\mathrm{R}$ package v.3.3.1. One-way ANOVA with post-hoc multiple comparison tests was performed using GraphPad Prism 8.2.1 software (GraphPad, La Jolla, USA). Error bars represent the relative standard deviation of the mean (RSD). Statistical significance was set at $P<$ 0.05 .

\section{Results and discussion Lipid identification}

The LC-MS/MS-based lipidomic analysis of lipid extracts (four replicates each) from whole adult female (Wf) worms of $H$. contortus and their reproductive (Rt) and alimentary (At) tracts allowed the identification and quantification of a total of 320 unique lipid species, belonging to four lipid categories (i.e. glycerolipids (GL), glycerophospholipids (GP), sphingolipids (SP) and sterol lipids (ST)) and 18 lipid classes (Table 1 and Additional file 1: Table S1). Consistent with the available global lipidome data set [11], lipid species from GL and GP categories were most commonly identified in all of the samples. Lipids from these two classes contributed to $\sim 90 \%$ of the lipid species identified, and TG was the most-commonly identified lipid class $(n=236)$, followed by glycerophosphoethanolamine (PE; $n=50$ ) and glycerophosphocholine (PC; $n=31$ ). By contrast, only small numbers of lipid species were found to represent the glycerophosphoglycerols (PG; $n=2$ ) and lyso-glycerophosphoglycerols (LPG; $n=3$ ) (Table 1 ). The number of lipid species shared among Rt, At and Wf samples are displayed in a Venn diagram (Fig. 1). Most of the lipid species $(n=296,92.5 \%)$ were commonly detected in at least two of the samples analysed. Most lipid species $(n$ $=245,76.6 \%)$ were detected in Wf, Rt and At, whereas 2, 10 and 12 lipid species were unique to each of these samples, respectively. The list of lipid species identified in individual samples is given in Additional file 1: Table S1.

\section{Lipid quantification}

Similar to the Wf lipidome [11], relative quantification analysis revealed that GP (49.6-82.1\%) and GL (16.1$49.8 \%$ ) were the two most abundant lipid categories in $H$. contortus at the organ system-level (i.e. in both Rt and At), whereas lipids from SP and ST categories contributed 
Table 1 Summary of the numbers of identified lipid species in extracts derived from whole adult female (Wf) worms of Haemonchus contortus and their reproductive (Rt) and alimentary (At) tracts

\begin{tabular}{|c|c|c|c|c|}
\hline Lipid category/class & Wf & Rt & At & $\begin{array}{l}\text { Total no. } \\
\text { of lipids } \\
\text { identified }\end{array}$ \\
\hline \multicolumn{5}{|l|}{ Glycerolipids } \\
\hline MG & 3 & 2 & 0 & 4 \\
\hline DG & 17 & 11 & 15 & 19 \\
\hline $\mathrm{TG}$ & 88 & 94 & 94 & 107 \\
\hline \multicolumn{5}{|l|}{ Glycerophospholipids } \\
\hline PC & 50 & 49 & 50 & 50 \\
\hline PE & 30 & 31 & 30 & 31 \\
\hline$P G$ & 2 & 2 & 2 & 2 \\
\hline $\mathrm{Pl}$ & 17 & 19 & 18 & 19 \\
\hline PS & 4 & 5 & 4 & 5 \\
\hline $\mathrm{CL}$ & 8 & 8 & 8 & 8 \\
\hline LPC & 15 & 16 & 16 & 16 \\
\hline LPE & 11 & 11 & 11 & 11 \\
\hline LPG & 3 & 2 & 2 & 3 \\
\hline LPI & 5 & 5 & 5 & 5 \\
\hline LPS & 7 & 7 & 7 & 7 \\
\hline \multicolumn{5}{|l|}{ Sphingolipids } \\
\hline SM & 4 & 4 & 3 & 4 \\
\hline Cer & 19 & 19 & 12 & 19 \\
\hline HexCer & 4 & 7 & 2 & 7 \\
\hline \multicolumn{5}{|l|}{ Sterol lipids } \\
\hline CE & 0 & 3 & 0 & 3 \\
\hline In total & 287 & 295 & 279 & 320 \\
\hline
\end{tabular}

Abbreviations: MG, monoradylglycerols; $\mathrm{DG}$, diradylglycerols;

TG, triradylglycerols; PC, glycerophosphocholines; $\mathrm{PE}$,

glycerophosphoethanolamines; PG, glycerophosphoglycerols; PI, glycerophosphoinositols; PS, glycerophosphoserines; CL, cardiolipins; SM, sphingomyelins; Cer, ceramide; HexCer, hexosylceramide; $\mathrm{CE}$, cholesteryl ester Note: For LPC, LPE, LPG, LPI and LPS, prefix "L" was added for each lysoglycerophospholipid class

$<2 \%$ to the total ion abundance in the lipidome of $H$. contortus (Fig. 2). Within the GP category, membrane structure-related PC (37-66\%) and PE (10.5-11.3\%) were the major classes. Notably, a significantly lower level of PC was measured in Rt compared with At (Fig. 2; Additional file 2: Table S2), although there was no significant difference for PE. Further analysis of individual lipid species showed that PC lipids with even-numbered fatty acyl chains (e.g. 18:0, 18:1 and 18:2) and long fatty acyl chains ( $>34$ total carbons) predominated (Fig. 3). In contrast, the energy storage-related TG (15.6-49.2\%) was the most abundant class in the GL category and had a significantly higher abundance in Rt than At (Fig. 4; Additional file 2: Table S2). Interestingly, TG species, such as TG (18:2_18:2_18:1) and TG (16:0_18:1_18:2), contributed

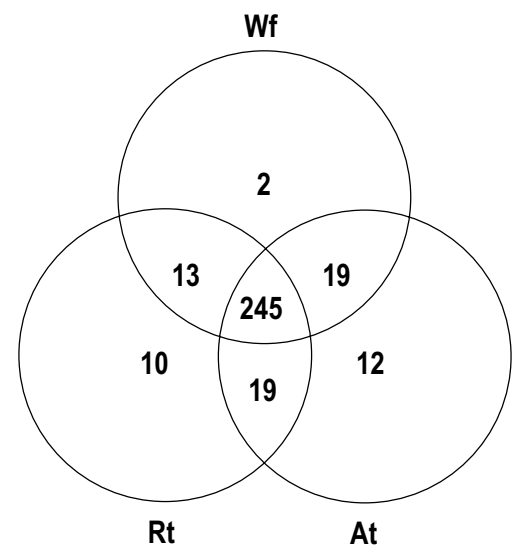

Fig. 1 Venn diagram showing the similarity of lipid species identified in extracts derived from whole adult female (Wf) worms of Haemonchus contortus and their reproductive (Rt) and alimentary (At) tracts

predominantly to this abundance difference between the two organ systems (tracts), whereas TG (18:1_18:1_18:1) did not. Similar to PC, individual lipid species with evennumbered fatty acyl chains (e.g. 18:1, 18:2 and 20:4) and long fatty acyl chain ( $>50$ total carbons)-fatty acids were the main representatives of the TG class. No significant difference in abundance was detected for lipid classes PG and LPG, both of which had a relatively low quantification level.

\section{Lipid composition}

The analysis of fatty acyl compositions showed that lipids of $H$. contortus contain a high level of unsaturated, evennumbered and long-chain fatty acyls (> 12 carbons), which represented $70.0 \%, 87.9 \%$ and $97.5 \%$ of the total fatty acyl composition, respectively (Table 2 ). The identified saturated lipid species were found mainly in ceramide (Cer, $n=8)$, hexosylceramide (HexCer, $n=4)$ and LPG $(n=4)$. In addition, 47 ether-linked lipid species were identified; they were mainly in the GP category ( $n$ $=42)$, representing particularly the classes $\mathrm{PE}(n=18)$ and PC $(n=9)$, whereas the other 5 ether-linked lipids represented the TG class. Nonetheless, no significant difference in the total ion abundance was detected for saturated or ether-linked lipids among three samples for each Wf, Rt and At (Fig. 5).

\section{Proposal that particular lipids are required for parasite adaptation, energy storage and development}

The constantly changing external environments force parasitic worms to adapt their nutrient acquisition and metabolism [21-23]. Previously, we observed substantial alterations in the lipidome of $H$. contortus, in terms of 
a

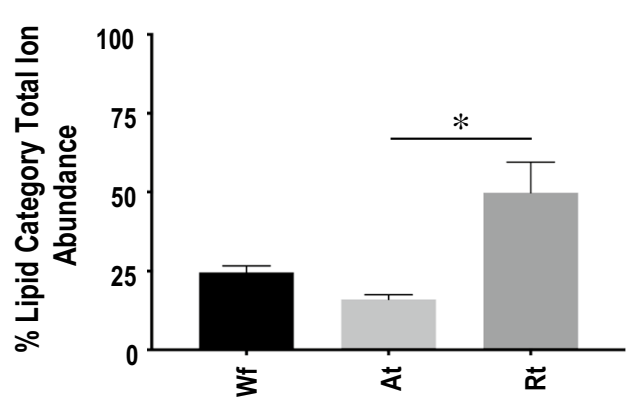

C

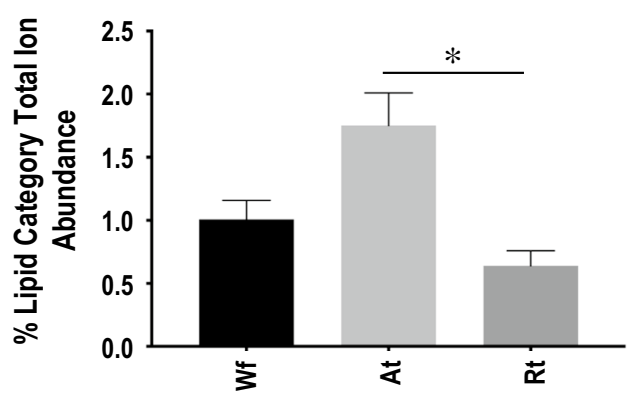

b

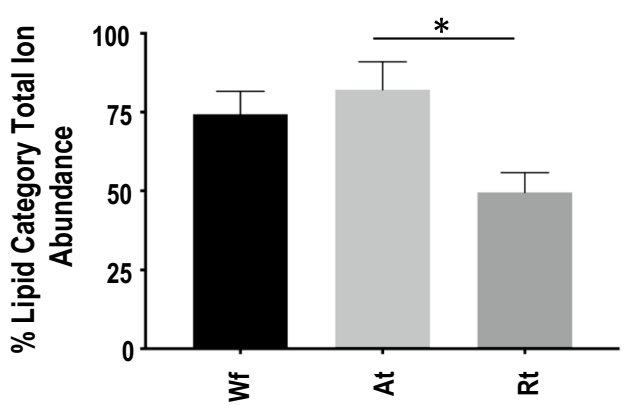

d

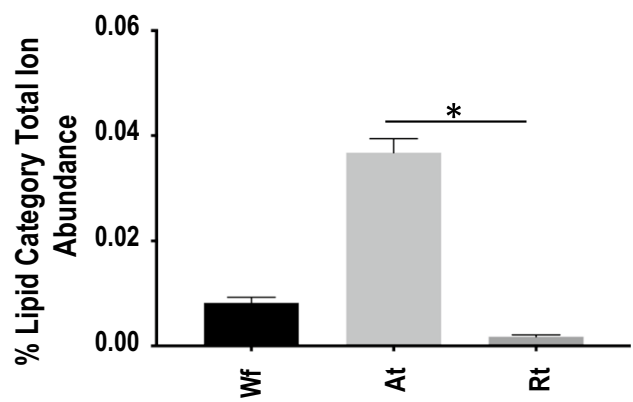

Fig. 2 Relative quantification changes of lipid categories from whole adult female (Wf) worms of Haemonchus contortus and their reproductive (Rt) and alimentary (At) tracts. a Glycerolipids. b Glycerophospholipids. c Sphingolipids. d Sterol lipids. Statistical analysis was performed by ANOVA $\left(^{*} P<\right.$ 0.05; see Additional file 2: Table S2 for details). Error bars indicate \pm RSD (four replicates)

lipid composition and abundance, during the transition from free-living to parasitic stages, indicating that energy metabolism was linked to adaptation in this nematode [11]. Conspicuously, the energy storage-related triradylglycerols (TG) abundance was relatively high in adult female worms of $H$. contortus.

The significantly higher abundance level of TG lipids in the reproductive system of the female worm compared with the gut indicated that the eggs in the uterus likely contribute most to the TG proportion in gravid female worms. Given that fatty acid beta-oxidation does not occur in the adult stage of parasitic worms of animals [21, 24], they are not able to consume exogenous lipids as a direct energy source. Evidence indicates that adult worms tend to modify (e.g. elongate and desaturate) as well as accumulate these incorporated fatty acids, mainly in the format of TG, into embryos for further development $[21,24]$. On the other hand, it has been shown that early egg and larval developmental stages of parasitic worms (e.g. H. contortus and Ascaris lumbricoides) can convert endogenous lipids acquired maternally from a previous host into carbohydrates (i.e. two acetyl-CoA units into succinate and malate) via beta-oxidation and glyoxylate cycle $[25,26]$. These endogenous lipids are believed to be an energy source (of predominantly TG) to prevent worm starvation and are building blocks (e.g. phospholipids) for the biosynthesis of membranes [24, 27-29]. Intriguingly, Bexkens et al. [24] observed a decrease in the number and size of lipid droplets (i.e. containing mainly TG) and an increase in cellular membrane lipids during egg development in the parasitic blood-fluke Schistosoma mansoni, suggesting that endogenous lipid stores are used for phospholipid biosynthesis. Based on a previous observation of lipid composition and abundance in eggs and third-stage larvae (L3s) of $H$. contortus [11], it is reasonable to propose that similar biosynthesis occurs during egg development in Haemonchus. It would be interesting to undertake a detailed 'time-course' lipidomic study of synchronised Haemonchus eggs during their development to larvae, in order to test this hypothesis.

\section{Parasite-derived lipids could play important roles in host-parasite interactions}

In addition to the biochemical roles of lipids in energy storage and membrane synthesis, it is becoming increasingly evident that parasite-derived lipids play important roles in host-parasite interactions, and that phospholipids (e.g. PC and PE) and lysophospholipids (e.g. LPC and LPS) are precursors for the regulation of various essential 

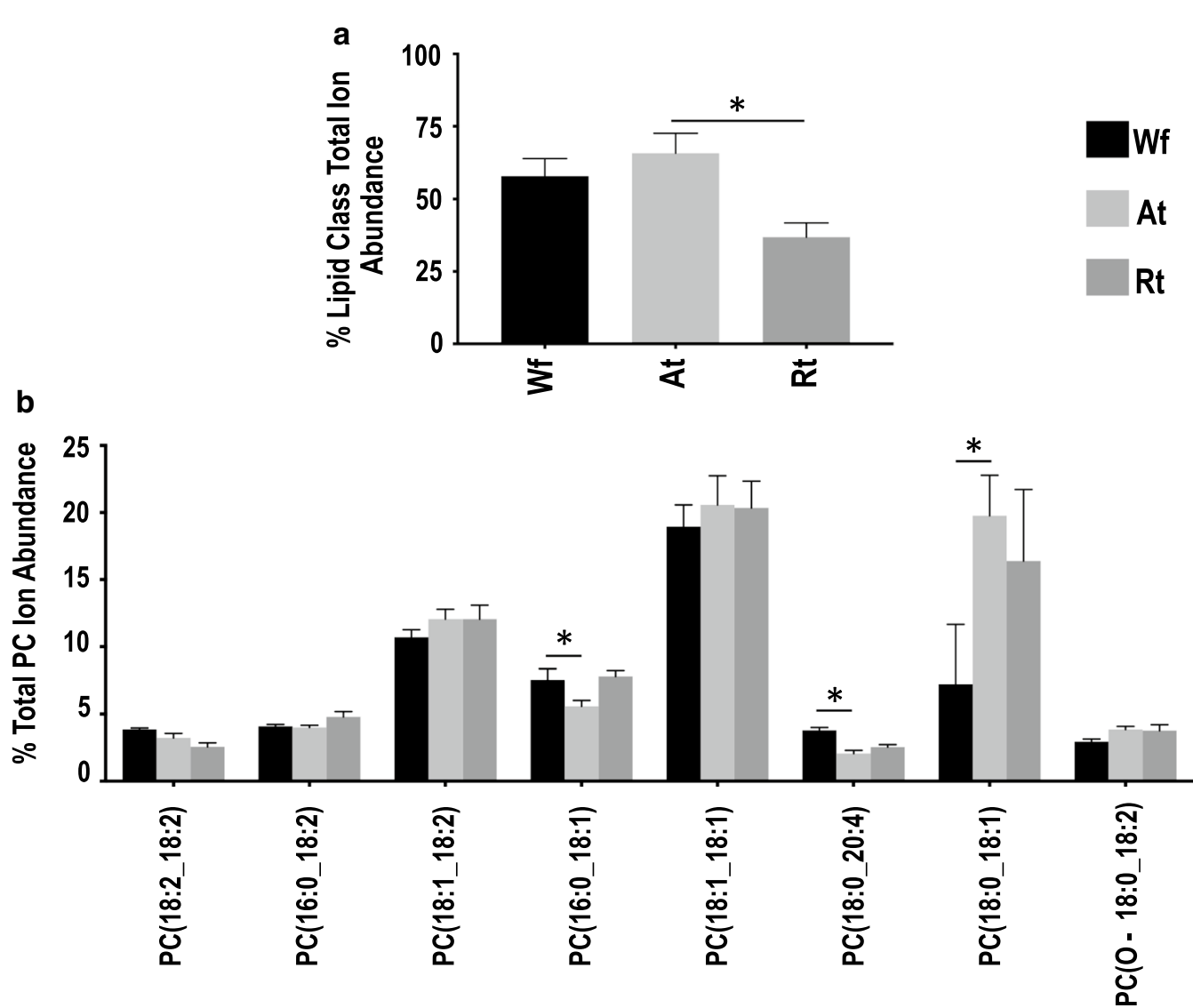

Fig. 3 Relative quantification changes of total (a) and individual (b) PC lipids from whole adult female (Wf) worms of Haemonchus contortus and their reproductive (Rt) and alimentary (At) tracts. Only individual lipid species with $>4 \%$ ion abundance (at any life stage) are shown. Statistical analysis was performed by ANOVA ( ${ }^{*}<<0.05$; see Additional file 2: Table S2 for details). Error bars indicate \pm RSD (four replicates)

signalling pathways in animals [30]. Previous biochemical studies of tegumental lipids of S. mansoni [27] have provided initial insight into the central roles that parasite-derived phospholipids and lysophospholipids play in lipid-associated host-parasite interactions. For instance, it has been shown that schistosome tegument-specific LPS and LPC species are able to downregulate host immune-modulation, leading to a decrease in eosinophil activation and cytokine production, via the stimulation of Toll-like receptor-2-dependent mechanisms in the immune system of the host animal [28, 31, 32]. Moreover, using direct infusion quadrupole time-of-flight (Q-TOF) mass spectrometry, some parasite-derived ether-linked phospholipids (i.e. PC and PE) were discovered in the fluid from Onchocerca ochengi-induced nodules in cattle [33], indicating a release of these molecules into host tissues. In the present study, substantial phospholipids (e.g. PC) were enriched in the gut of $H$. contortus, including ether-linked phospholipids (e.g. PC E-34:2, PC E-36:2 and PC E-38:2) (Fig. 3 and Additional file: Table S1). Unlike the lipid-enriched tegument of flatworms, which transports nutrients into the worm and by-products out of the worm [27], the surface of parasitic nematodes is covered by a tough and almost impermeable cuticle, composed primarily of proteins and a limited amount of lipids [34]. This means that the nematode intestine is likely the primary source of worm-derived lipids, released for cross-talk between worm and host. Although there is presently no direct evidence, it seems reasonable to propose that nematode-derived glycerophospholipids are released directly from nematode intestine, possibly requiring lipid transfer or transport proteins. Interestingly, in a previous study of the developmental secretome of $H$. contortus, we identified a panel of lipid binding-associated proteins $(n=8)$, such as fatty acid and retinoid-binding proteins and vitellogenin (egg-yolk protein) [10]. These proteins were believed to be involved in lipid transportation [34]. Evidence that such proteins are excreted/secreted mainly by parasitic stages (i.e. L4, adult female and adult male), rather than free-living L3 stage (Fig. 6), suggests heavy demands on the use of these proteins as vehicles for worm-derived lipid delivery to 

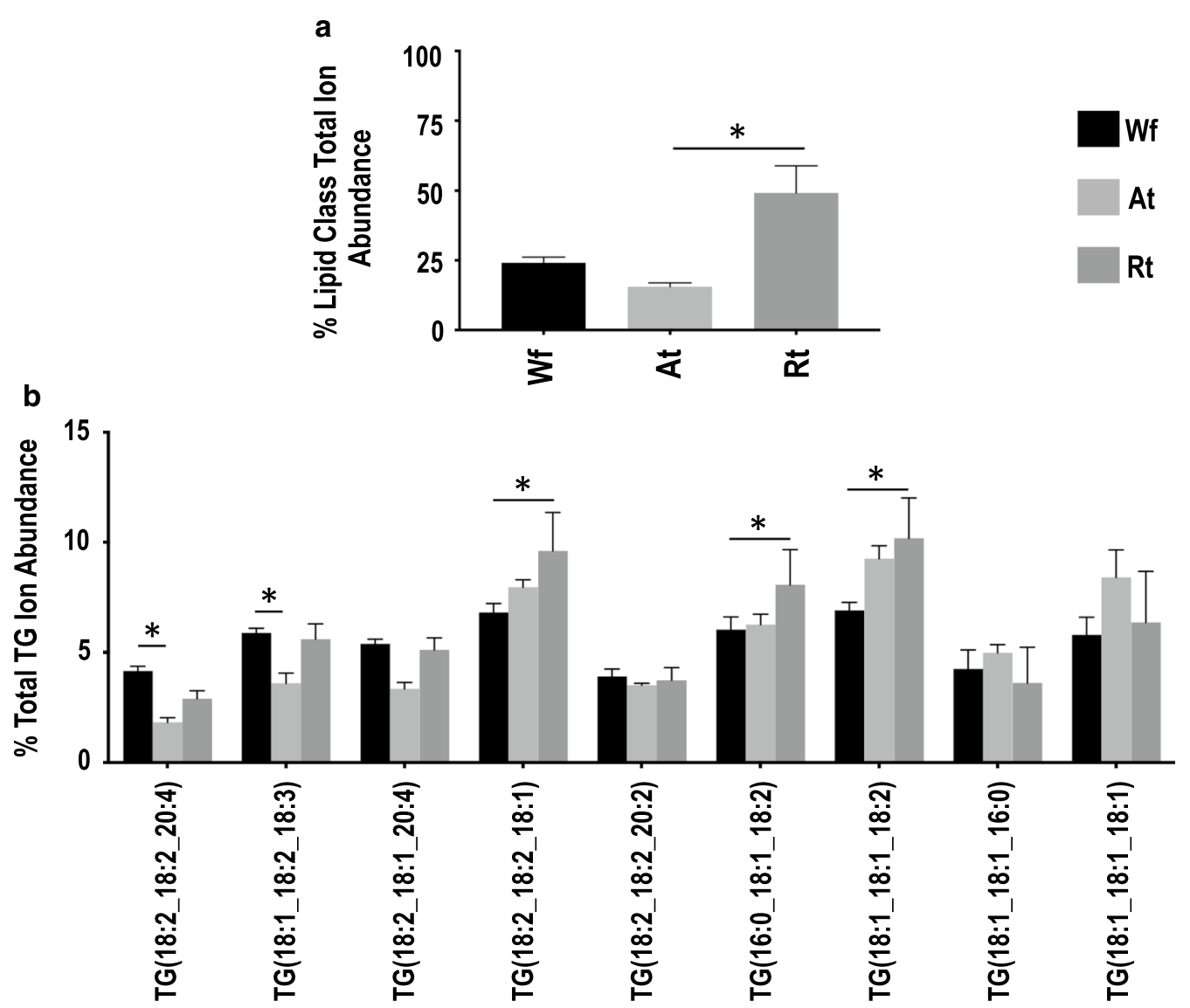

Fig. 4 Relative quantification changes of total (a) and individual (b) TG lipids from whole adult female (Wf) worms of Haemonchus contortus and their reproductive (Rt) and alimentary (At) tracts. Only individual lipid species with $>4 \%$ ion abundance are shown. Statistical analysis was performed by ANOVA ( ${ }^{*} P<0.05$; see Additional file 2: Table $S 2$ for details). Error bars indicate \pm RSD (four replicates)

Table 2 Fatty acyl (FA) composition of identified lipid species in the lipidome of the adult female of Haemonchus contortus

\begin{tabular}{|c|c|c|c|c|c|c|c|}
\hline \multirow[t]{2}{*}{ Lipid category } & \multicolumn{2}{|c|}{ Saturated (\%) } & \multicolumn{2}{|c|}{ Unsaturated (\%) } & \multirow{2}{*}{$\begin{array}{l}\text { Odd-numbered } \\
\text { chain FA (\%) }\end{array}$} & \multirow{2}{*}{$\begin{array}{l}\text { Even-numbered } \\
\text { chain FA (\%) }\end{array}$} & \multirow[t]{2}{*}{ Total no. of FAs } \\
\hline & $\begin{array}{l}\text { Medium- } \\
\text { chain FA }\end{array}$ & Long-chain FA ${ }^{b}$ & $\begin{array}{l}\text { Medium- } \\
\text { chain FA }\end{array}$ & Long-chain FA & & & \\
\hline Glycerolipids & 2.5 & 11.4 & nd & 37.0 & 5.2 & 45.7 & 796 \\
\hline Glycerophospholipids & nd & 10.9 & nd & 29.3 & 2.2 & 38.0 & 413 \\
\hline Sphingolipids & nd & 5.2 & nd & 3.2 & 4.6 & 3.8 & 78 \\
\hline Steroid lipids & nd & 0 & nd & 0.4 & nd & 0.4 & 11 \\
\hline In total & 2.5 & 27.5 & 0 & 70.0 & 12.1 & 87.9 & 1298 \\
\hline
\end{tabular}

a Medium-chain FA contains 6-12 carbons

${ }^{\mathrm{b}}$ Long-chain FA contains $>12$ carbons

Abbreviation: nd, not detected 
a

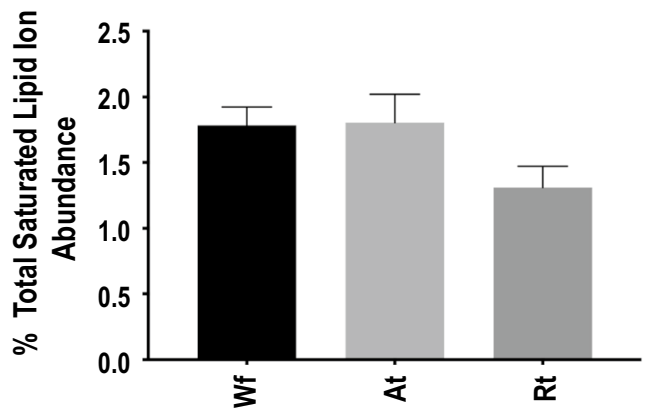

b

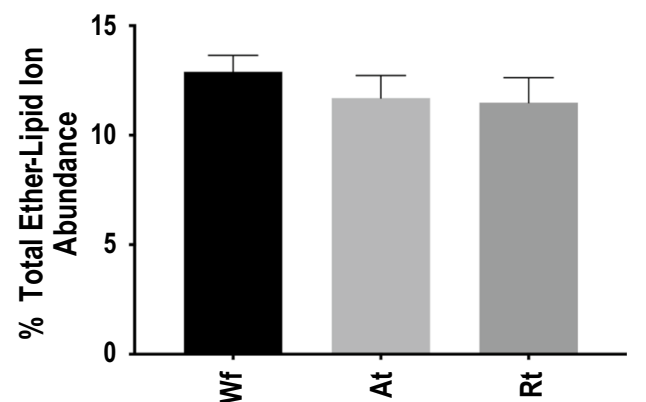

Fig. 5 Relative quantification changes of (a) total saturated fatty acyl- and (b) ether-linked lipid ion abundance in the whole adult female (Wf) worms of Haemonchus contortus and their reproductive (Rt) and alimentary (At) tracts. Error bars indicate \pm RSD (four replicates)

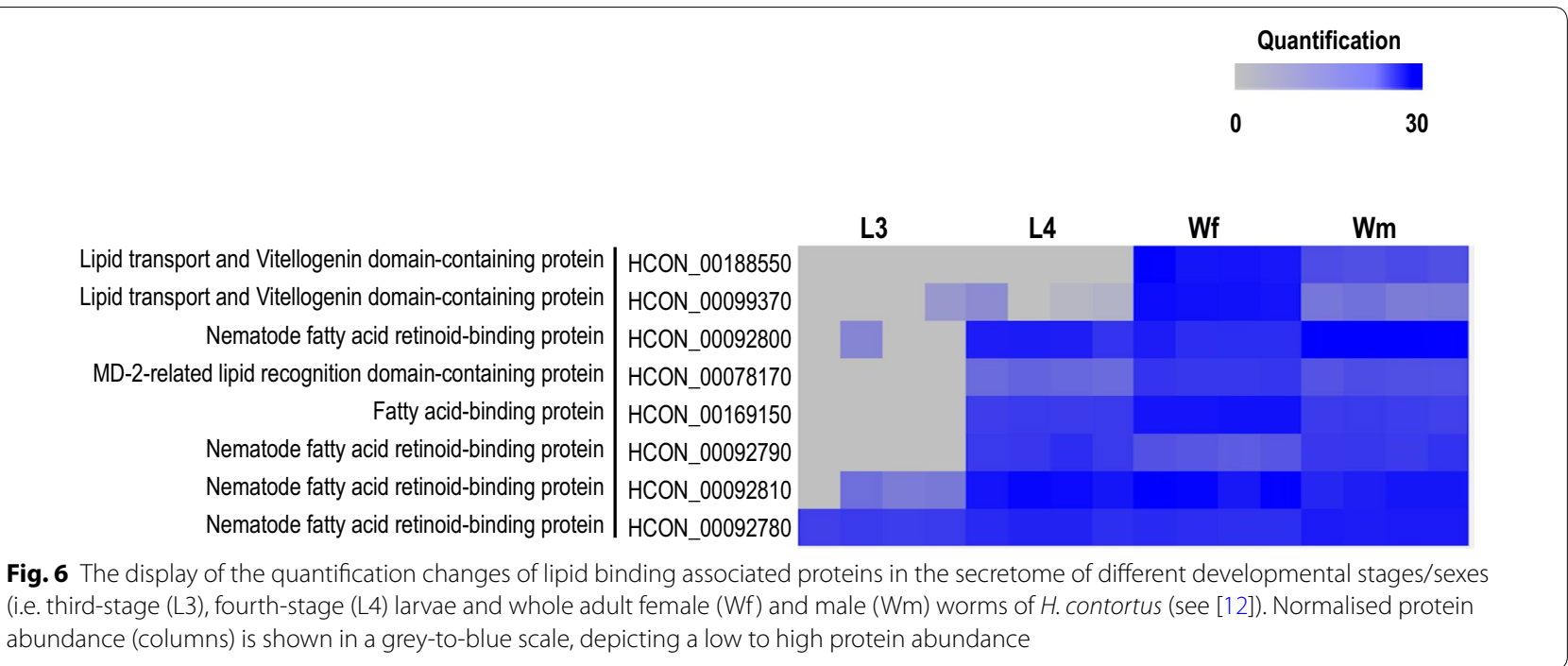

the host as Haemonchus establishes in the stomach (abomasum) of its host animal.

\section{Conclusions}

This high-throughput LC-MS/MS-based lipidomic study has inferred that a spectrum of $H$. contortus lipids is likely required as an energy source, for development and adaptation and/or for the maintenance of an intricate host-parasite cross-talk. Finding ways of disrupting or interrupting this interplay has the potential to lead to new intervention strategies.

\section{Supplementary information}

Supplementary information accompanies this paper at https://doi. org/10.1186/s13071-020-04208-w.

Additional file 1: Table S1. Relative quantification changes of total saturated fatty acids and ether-linked lipid ion abundance from whole adult female (Wf) worms of Haemonchus contortus and their reproductive (Rt) and alimentary (At) tracts.

Additional file 2: Table S2. Results of statistical analyses (one-way ANOVAs).

\section{Abbreviations}

Wf: whole adult female; Rt: reproductive tract; At: alimentary track; MG: monoradylglycerols; DG: diradylglycerols; TG: triradylglycerols; PC: 
glycerophosphocholines; PE: glycerophosphoethanolamines; PG: glycerophosphoglycerols; PI: glycerophosphoinositols; PS: glycerophosphoserines; CL: cardiolipins; For LPC, LPE, LPG, LPI and LPS, prefix "L" was added for each lysoglycerophospholipid class; SM: sphingomyelins; Cer: ceramide; HexCer: hexosylceramide; CE: cholesteryl ester; FA: fatty acyl; RSD: relative standard deviation.

\section{Acknowledgements}

We would like to thank Mr Yepy Hardi Rustam (Department of Biochemistry and Molecular Biology, The University of Melbourne) for his kind support with lipid extraction.

\section{Authors' contributions}

TW and RBG planned the study. TW, GM and SN undertook the laboratory work. TW analysed the results. TW and RBG wrote the manuscript with inputs from GM, SN, NAW and GER. RBG and GER raised the funds for the project. All authors read and approved the final manuscript.

\section{Funding}

The present study was funded by the Australian Research Council (ARC) and The University of Melbourne. The funding body played no role in the design of the study or sample collection, data analysis or interpretation, or in the writing of the manuscript.

\section{Availability of data and materials}

Data supporting the conclusions of this article are included within the article.

\section{Ethics approval and consent to participate}

The animal ethical approval of sample collection was obtained from the University of Melbourne (permit no. 1714374).

\section{Consent for publication}

Not applicable.

\section{Competing interests}

The authors declare that they have no competing interests.

\section{Author details}

${ }_{1}^{1}$ Department of Veterinary Biosciences, Melbourne Veterinary School, Faculty of Veterinary and Agricultural Sciences, The University of Melbourne, Parkville, Victoria 3010, Australia. ${ }^{2}$ Bio21 Mass Spectrometry and Proteomics Facility, The University of Melbourne, Parkville, Victoria 3010, Australia. ${ }^{3}$ School of Chemistry, The University of Melbourne, Parkville, Victoria 3010, Australia. ${ }^{4}$ Department of Biochemistry and Molecular Biology, The University of Melbourne, Parkville, Victoria 3010, Australia. ${ }^{5}$ Bio21 Molecular Science and Biotechnology Institute, The University of Melbourne, Parkville, Victoria 3010, Australia.

Received: 20 May 2020 Accepted: 26 June 2020

Published online: 06 July 2020

\section{References}

1. Gasser RB, von Samson-Himmelstjerna G. Haemonchus contortus and haemonchosis - past, present and future trends. Adv Parasitol. 2016;93:1-665.

2. Priority list of endemic diseases for the red meat industries. https://www. mla.com.au/research-and-development/search-rd-reports/final-report-detai Is/Animal-Health-and-Biosecurity/Priority-list-of-endemic-diseases-for-thered-meat-industries/2895. Accessed 1 Apr 2020.

3. Besier RB, Kahn LP, Sargison ND, Van Wyk JA. Diagnosis, treatment and management of Haemonchus contortus in small ruminants. Adv Parasitol. 2016:93:181-238

4. Kotze AC, Prichard RK. Anthelmintic resistance in Haemonchus contortus: history, mechanisms and diagnosis. Adv Parasitol. 2016;93:397-428.

5. Mederos AE, Ramos Z, Banchero GE. First report of monepantel Haemonchus contortus resistance on sheep farms in Uruguay. Parasit Vectors. 2014;7:598.

6. Doyle SR, Laing R, Bartley DJ, Britton C, Chaudhry U, Gilleard JS, et al. A genome resequencing-based genetic map reveals the recombination landscape of an outbred parasitic nematode in the presence of polyploidy and polyandry. Genome Biol Evol. 2018;10:396-409.
7. Laing R, Kikuchi T, Martinelli A, Tsai IJ, Beech RN, Redman E, et al. The genome and transcriptome of Haemonchus contortus, a key model parasite for drug and vaccine discovery. Genome Biol. 2013;14:R88.

8. Schwarz EM, Korhonen PK, Campbell BE, Young ND, Jex AR, Jabbar A, et al. The genome and developmental transcriptome of the strongylid nematode Haemonchus contortus. Genome Biol. 2013;14:R89.

9. Ma G, Wang T, Korhonen PK, Ang CS, Williamson NA, Young ND, et al. Molecular alterations during larval development of Haemonchus contortus in vitro are under tight post-transcriptional control. Int J Parasitol. 2018;48:763-72.

10. Wang T, Ma G, Ang CS, Korhonen PK, Koehler AV, Young ND, et al. High throughput LC-MS/MS-based proteomic analysis of excretory-secretory products from short-term in vitro culture of Haemonchus contortus. J Proteomics. 2019;204:103375.

11. Wang T, Nie S, Ma G, Korhonen PK, Koehler AV, Ang CS, et al. The developmental lipidome of Haemonchus contortus. Int J Parasitol. 2018;48:887-95.

12. Wang T, Ma G, Ang CS, Korhonen PK, Rong X, Nie S, et al. Somatic proteome of Haemonchus contortus. Int J Parasitol. 2019;49:311-20.

13. Wang T, Ma G, Ang CS, Korhonen PK, Stroehlein AJ, Young ND, et al. The developmental phosphoproteome of Haemonchus contortus. J Proteomics. 2020;213:103615.

14. Ma G, Wang T, Korhonen PK, Young ND, Nie S, Ang CS, et al. Dafachronic acid promotes larval development in Haemonchus contortus by modulating dauer signalling and lipid metabolism. PLoS Pathog. 2019;15:e1007960.

15. Barrett J, Ward CW, Fairbairn D. The glyoxylate cycle and the conversion of triglycerides to carbohydrates in developing eggs of Ascaris lumbricoides. Comp Biochem Phys. 1970;35:577-86.

16. Page AP, Johnstone IL. The cuticle (March 19, 2007). In: The C. elegans Research Community, editor. WormBook; 2007. http://www.wormbook.org.

17. Folch J, Lees $M$, Sloane Stanley GH. A simple method for the isolation and purification of total lipides from animal tissues. J Biol Chem. 1957:226:497-509.

18. Narvaez-Rivas M, Zhang Q. Comprehensive untargeted lipidomic analysis using core-shell C30 particle column and high field orbitrap mass spectrometer. J Chromatogr A. 2016;1440:123-34.

19. Liebisch G, Vizcaino JA, Kofeler H, Trotzmuller M, Griffiths WJ, Schmitz G, et al, Shorthand notation for lipid structures derived from mass spectrometry. J Lipid Res. 2013;54:1523-30.

20. Ryan E, Reid GE. Chemical derivatization and ultrahigh resolution and accurate mass spectrometry strategies for "shotgun"lipidome analysis. Acc Chem Res. 2016:49:1596-604.

21. Campbell BE, Nagaraj SH, Hu M, Zhong W, Sternberg PW, Ong EK, et al. Gender-enriched transcripts in Haemonchus contortus - predicted functions and genetic interactions based on comparative analyses with Caenorhabditis elegans. Int J Parasitol. 2008;38:65-83.

22. Harder A. The biochemistry of Haemonchus contortus and other parasitic nematodes. Adv Parasitol. 2016:93:69-94.

23. Armstrong SD, Xia D, Bah GS, Krishna R, Ngangyung HF, LaCourse EJ, et al. Stage-specific proteomes from Onchocerca ochengi, sister species of the human river blindness parasite, uncover adaptations to a nodular lifestyle. Mol Cell Proteomics. 2016;15:2554-75.

24. Wang T, Van Steendam K, Dhaenens M, Vlaminck J, Deforce D, Jex AR, et al. Proteomic analysis of the excretory-secretory products from larval stages of Ascaris suum reveals high abundance of glycosyl hydrolases. PLoS Negl Trop Dis. 2013;7:e2467.

25. Bexkens ML, Mebius MM, Houweling M, Brouwers JF, Tielens AGM, van Hellemond JJ. Schistosoma mansoni does not and cannot oxidise fatty acids, but these are used for biosynthetic purposes instead. Int J Parasitol. 2019;49:647-56.

26. Carrington M, Jasmer D, McFadden B. Activities of isocitrate lyase and malate synthase during the development of free-living stages Haemonchus contortus (Nematoda). Proc Helminth Soc Wash. 1987;54:277-9.

27. Ward CW, Fairbairn D. Enzymes of beta-oxidation and their function during development of Ascaris lumbricoides eggs. Dev Biol. 1970;22:366-87.

28. Van Hellemond JJ, Retra K, Brouwers JF, van Balkom BW, Yazdanbakhsh M, Shoemaker CB, Tielens AG. Functions of the tegument of schistosomes: clues from the proteome and lipidome. Int J Parasitol. 2006;36:691-9.

29. Retra K, deWalick S, Schmitz M, Yazdanbakhsh M, Tielens AG, Brouwers JF, van Hellemond JJ. The tegumental surface membranes of Schistosoma mansoni are enriched in parasite-specific phospholipid species. Int J Parasitol. 2015;45:629-36. 
30. Becker A, Willenberg I, Springera A, Schebb NH, Steinberg P, Strub C. Fatty acid composition of free-living and parasitic stages of the bovine lungworm Dictyocaulus viviparus. Mol Biochem Parasitol. 2017;216:39-44

31. Watts JL, Ristow M. Lipid and carbohydrate metabolism in Caenorhabditis elegans. Genetics. 2017;207:413-46.

32. Van der Kleij D, Latz E, Brouwers JF, Kruize YC, Schmitz M, Kurt-Jones EA, et al. A novel host-parasite lipid cross-talk. Schistosomal lyso-phosphatidylserine activates toll-like receptor 2 and affects immune polarization. J Biol Chem. 2002;277:48122-9.

33. Magalhaes K, Almeida PE, Atella G, Maya-Monteiro CM, Castro-Faria-Neto H, Pelajo-Machado M, et al. Schistosomal-derived lysophosphatidylcholine are involved in eosinophil activation and recruitment through Toll-like receptor2-dependent mechanisms. J Infect Dis. 2010;202:1369-79.

34. Wewer V, Makepeace BL, Tanya VN, Peisker H, Pfarr K, Hoerauf A, Dormann P. Lipid profiling of the filarial nematodes Onchocerca volvulus, Onchocerca ochengi and Litomosoides sigmodontis reveals the accumulation of nematode-specific ether phospholipids in the host. Int J Parasitol. 2017:47:903-12.

\section{Publisher's Note}

Springer Nature remains neutral with regard to jurisdictional claims in published maps and institutional affiliations.
Ready to submit your research? Choose BMC and benefit from:

- fast, convenient online submission

- thorough peer review by experienced researchers in your field

- rapid publication on acceptance

- support for research data, including large and complex data types

- gold Open Access which fosters wider collaboration and increased citations

- maximum visibility for your research: over $100 \mathrm{M}$ website views per year

At BMC, research is always in progress.

Learn more biomedcentral.com/submissions 